Images du travail, travail des images

4 | 2017

La relation soignants/soignés à l'épreuve de l'image

Bloncourt G. (2016), L'œil en colère, journalisme et révolution, Paris, Lemieux éditeur

Henri Eckert

\title{
OpenEdition
}

Journals

Édition électronique

URL : http://journals.openedition.org/itti/1045

DOI : 10.4000/itti. 1045

Éditeur

Université de Poitiers

Référence électronique

Henri Eckert, «Bloncourt G. (2016), L'œil en colère, journalisme et révolution, Paris, Lemieux éditeur »,

Images du travail, travail des images [En ligne], 4 | 2017, mis en ligne le 01 septembre 2017, consulté le 14 avril 2021. URL : http://journals.openedition.org/itti/1045 ; DOI : https://doi.org/10.4000/itti.1045

Ce document a été généré automatiquement le 14 avril 2021

Images du travail, travail des images 


\section{Bloncourt G. (2016), L'œil en colère, journalisme et révolution, Paris, Lemieux éditeur}

Henri Eckert

RÉFÉRENCE

Bloncourt G. (2016), L'œil en colère, journalisme et révolution, Paris, Lemieux éditeur

Sur le front du travail 


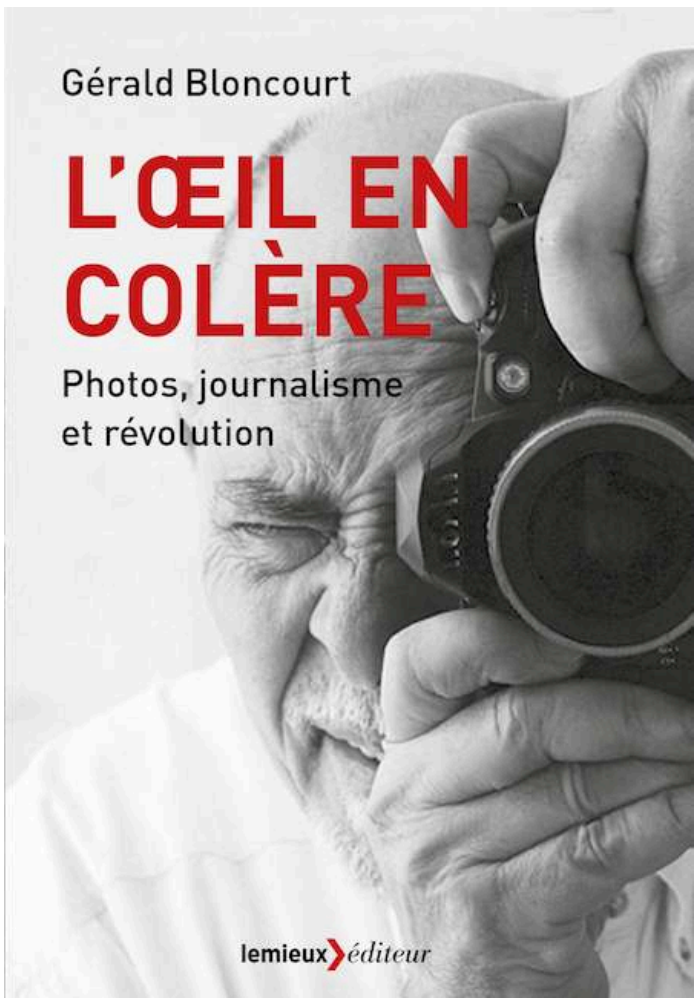

1 Ce petit livre de Gérald Bloncourt - L'œil en colère - intéressera sans doute ses lecteurs parce qu'il se donne d'emblée comme une autobiographie et, d'une certaine manière, comme un testament: celui d'un photojournaliste engagé dans les luttes politiques et sociales de son époque. Mais il les intéressera sans doute aussi - je dirais : dans l'élan d'un récit toujours ancré dans l'épaisseur des pratiques, sociales ou professionnelles parce qu'il leur propose simultanément, de manière étroitement imbriquée à la vie de l'auteur, une description précise du métier de photojournaliste, voire, plus précisément encore, une plongée dans le travail effectif, concret d'un photojournaliste.

Gérald Bloncourt n'écrit-il pas, dès la première page du livre, après avoir évoqué les glorieux pionniers de la pratique photographique, Niepce, Daguerre ou encore Nadar :

"Comme eux, je me suis servi des mêmes outils, des mêmes crémaillères en laiton, des mêmes soufflets en cuir. Comme eux, j'ai effectué des gestes identiques pour mettre en place la grosse chambre 18x24 ou manœuvré l'obturateur à l'aide d'une poire. Comme eux, je me suis mis la tête sous l'épais voile noir et j'ai utilisé les mêmes plaques sensibles en verre. » (p. 9)

Et de raconter un peu plus loin comment il a photographié les coureurs du Tour de France pour le compte de L'Humanité, « derrière moto ", c'est-à-dire assis à l'arrière d'une moto, en équilibre instable manœuvrant en la circonstance un appareil Gaumont comportant deux magasins qui abritaient des plaques de verre aux formats $9 \times 12$ ou $6 \times 9$, certes plus petit que les chambres d'antan et équipé, précise-t-il, d'objectifs de 50 à 90 millimètres de focale. Les zooms étaient alors rares, tout comme les téléobjectifs et Gérald Bloncourt raconte comment il découvre, quelques années plus tard, l'existence de ces fameux objectifs qui rapprochent l'objet photographié. À l'occasion d'un incendie sur la Tour Eiffel, alors que les photographes locaux s'étaient, comme luimême, hissés péniblement sur la plate-forme de la tour pour réaliser leurs clichés, ils 
découvrent après en être redescendus, de l'autre côté de la Seine, trois reporters américains regroupés autour d'un téléobjectif « de près de deux mètres » :

« Nous leur demandons la permission de jeter un coup d'œil dans le viseur. À la queue leu leu, chacun de nous peut se rendre compte du prodigieux effet. Nous ne sommes qu'à deux mètres de l'endroit ! Nous pouvons presque toucher les pompiers qui s'affairent encore. » (p. 86)

Il évoque de même les appareils qu'il a manipulés, du Rolleiflex avec lequel il réalise de nombreux portraits de célébrités au boîtier numérique, en passant par le Mamiya 6x7, un appareil exceptionnel dit-il.

5 Il serait vain, assurément, de prétendre ignorer les conditions techniques d'exercice du métier de photographe, de négliger les contraintes induites par les outils de travail, qu'il s'agisse du boîtier ou des optiques qui l'équipent, ou les conséquences des modes d'enregistrement de l'image, qu'elle ait été saisie sur un film argentique ou, plus récemment, par un capteur numérique - Gérald Bloncourt rapporte dans quelles circonstances il est, selon la formule, passé au numérique ${ }^{1}$. Mais s'il prend soin de faire ainsi état des effets des techniques sur la pratique du métier de photographe, l'intérêt de ses propos sur l'exercice du métier réside, davantage encore, dans l'attention qu'il porte à la description des circonstances dans lesquelles il a produit ses photographies et à la spécificité de sa pratique, lorsqu'il évoque, notamment, les rapports qu'il a entretenus avec les individus qu'il photographie. «Ma façon de travailler, c'est de vivre au milieu de ceux que je photographie » (p. 136) écrit-il. Il déclarait d'ailleurs, quelques pages plus tôt :

«Je parcours la France des conflits sociaux. Les usines, les mines, les ports, les chantiers du bâtiment... Je vis avec les travailleurs. Je partage leurs repas. Je photographie leurs gosses dans les corons, leurs femmes qui se mobilisent lorsqu'ils sont en grève, les manifestations qui ont lieu dans leurs villages... » (p. 127)

Et d'ajouter, comme en conclusion de cette revendication d'une pratique du photojournalisme qui s'appuie sur une immersion dans la vie des personnes qu'il photographie :

«Je compose mes photos comme des tableaux en situant chaque élément du décor.»

7 Gérald Bloncourt avait, il est vrai, prévenu son lecteur depuis longtemps, juste après avoir évoqué sa rencontre fortuite avec Robert Capa :

«Puisque Capa allait sur les théâtres d'opération pour mieux combattre la guerre,

j'irai dans les usines pour dénoncer la misère. » (p. 39)

Si ses photos s'inscrivent ainsi dans une réalité qu'il connaît bien pour avoir vécu avec les femmes ou les hommes qui s'y confrontent, l'engagement dont elles témoignent ne l'empêche pas de composer ses images "comme des tableaux", avec le souci de les construire sans renoncer à l'exactitude et à la fidélité aux contextes des vies qu'elles révèlent. Sans éluder la colère de ces hommes aussi : "Assez de brimades - De la dignité " sont les mots que rapporte une photo réalisée par Gérald Bloncourt, prise dans une allée de l'usine Renauld de Billancourt, lors des grandes grèves de 1968, alors que le photographe a passé trente-trois jours avec les ouvriers qui occupaient l'usine. 
Image 2

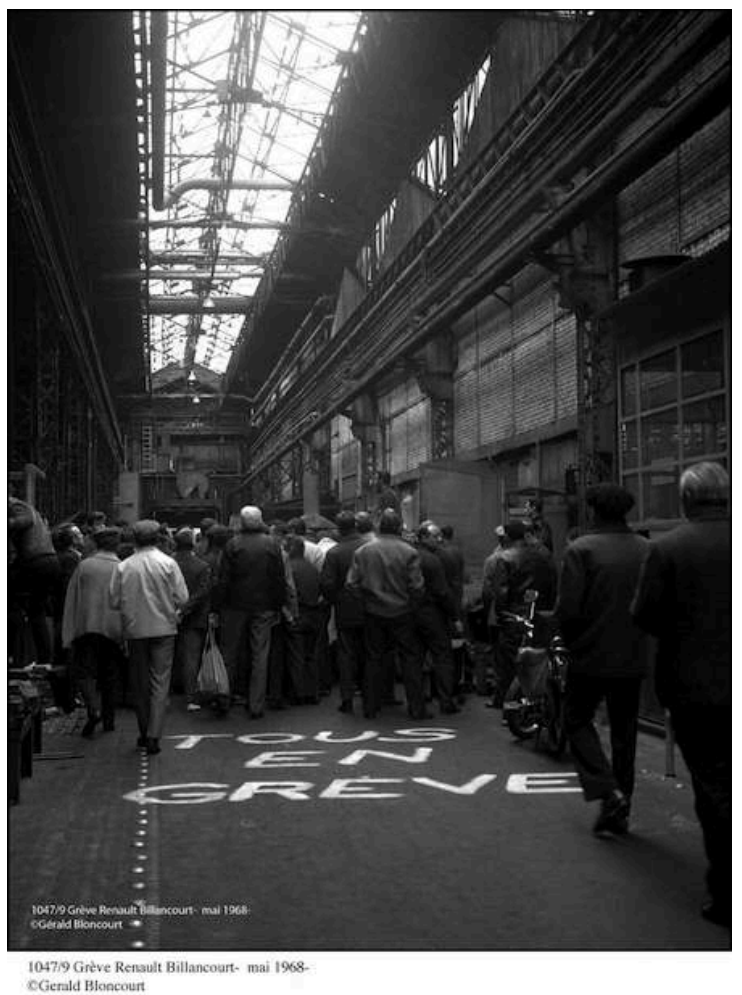

9 Cette pratique engagée a pris davantage consistance encore à partir du moment où Gérald Bloncourt cesse de travailler exclusivement, en tant que salarié, pour le compte de journaux du Parti communiste. Critique par rapport à la ligne officielle du parti, il est d'abord éloigné du service photographique de L'Humanité avant d'être tout bonnement remercié par la direction de L'avant-garde qui l'avait accueilli. Mais pas question pour lui de « collaborer avec la presse "bourgeoise" » (p. 123) ; c'est désormais la presse syndicale qui va lui permettre de poursuivre son travail de photojournaliste, en tant que pigiste. Il y trouve l'occasion d'affirmer plus encore une pratique qui revendique un autre rapport aux sujets et aux objets qu'il photographie. Gérald Bloncourt a rappelé, dès le début du livre, comment il s'est « rapidement trouvé en porte-à-faux » avec la façon dont la rédaction de L'Huma envisageait la photo pour rendre compte des manifestations ou des grèves ouvrières.

«Pour la rédaction de L'Huma, il fallait regrouper les travailleurs, les aligner en rangs d'oignons devant un chantier, les camper bien droit devant leurs usines. » (p. 31)

Gérald Bloncourt sent qu'il faut faire autre chose :

«Je sens qu'il faut photographier la vie telle qu'elle se présente, sans fard, sans intervenir en la recomposant. Il est plus fort, plus vrai, de saisir ces hommes tels qu'on les voit, avec leurs gestes et leur colère. » (p. 31)

Lors des grandes grèves du mois d'août 1953, Gérald Bloncourt présente « une photo d'une palissade où est écrit à la craie, d'une main rageuse : "Chantier en grève de 24 heures" ", photo que L'Huma ne publiera en une qu'après d'âpres discussions, tant la photo rompait avec les codes en vigueur! Dix ans plus tard, alors qu'il ne travaille plus pour L'Huma, Gérald Bloncourt radicalise son point de vue. Il couvre une autre grande grève, celle des « gueules jaunes » de Trieux, dans le bassin de Longwy : 
«Ils décident d'occuper la mine. Dans toute la région, la solidarité s'organise. Tous les jours, un meeting sur la place. Un des mineurs amène chaque fois sa petite fille (elle doit avoir cinq ans) avec lui. Elle lui tient la main et cherche à se cacher derrière lorsque nos regards se croisent. Au bout du troisième jour, l'évidence s'impose : c'est elle "la grève", c'est son avenir que son père défend ! »²

La photo sera publiée en pleine page par La vie ouvrière, avec cette légende : «Trieux 1963 - La grève!» Gérald Bloncourt ne cesse, du reste, d'insister sur l'enjeu de la légende qui accompagne généralement toute photographie, au point même de souligner, paradoxalement, "combien une photo qui ne donne pratiquement rien à voir peut, avec ce que suggère sa légende, retenir l'attention et être en fin de compte publiée... » (p. 141)

13 S'immerger ainsi dans un milieu, côtoyer les protagonistes, participer à leur vie ordinaire - quasiment à la manière du sociologue qui s'engage dans une démarche ethnographique - et prendre des photos ne va pourtant pas tout à fait de soi. L'expression " prendre une photo » suggère la difficulté :

"Je me suis souvent questionné sur le fait d'avoir été un "voyeur" insatiable, m'arrogeant le droit de m'approprier l'âme d'individus sans leur demander leur accord ou leur avis pour les donner à voir à des milliers d'autres. De mettre à nu leur détresse, leur pauvreté et quelques fois leurs larmes. De quel droit? » (p. 206)

Et de poursuivre: «Je me suis rassuré en me persuadant que j'étais un témoin de l'Histoire. » (p. 206) Et, plus loin encore :

«J'ai photographié des millions de travailleuses et de travailleurs dans leur vie quotidienne. J'ai vécu leurs luttes, pied à pied, contre leurs exploiteurs. Ils venaient de tous les coins du monde. Avaient toutes les religions de la terre. Toutes les opinions politiques. Mes images ont montré leur humanité, leur dignité. Je n'ai pas cherché à savoir s'ils votaient rouge, rose ou autre chose... Avec mon objectif, je les ai montrés, créateurs de richesses, défendant leurs droits. J'ai voulu écrire l'histoire de ceux qui veulent un autre monde, d'autres conditions de vie... La photographie m'a permis de véhiculer leur colère, leur désespoir, leurs certitudes, leurs sourires, leur beauté, leur grandeur... »

Et puis quoi, il y a aussi cette fillette de Saint-Denis : voilà pas mal de temps que Gérald Bloncourt arpente le bidonville des Portugais de Saint-Denis ; un soir il y croise Maria ${ }^{3}$, qui avait repéré le photographe et qui avait bien envie d'être prise en photo!

Il y aurait bien d'autres passages du livre à évoquer. Le chapitre dans lequel Gérald Bloncourt rapporte la création, à l'initiative de Roger Pic, de l'Association nationale des journalistes reporters photographes et cinéastes (ANJRPC), à laquelle lui-même prend part, association professionnelle chargée de défendre les intérêts spécifiques des photographes notamment. Ou alors, dans un autre registre, les ruses du photographe parti à Nice sur un tournage de film pour en rapporter une photo de Jean-Paul Belmondo et Jeanne Moreau. Le livre fourmille d'anecdotes, graves ou plus légères. Mais il donne, en même temps que l'itinéraire d'un photographe engagé dans les luttes de son temps, une idée du métier de photographe, de son travail et des questions que pose cette étrange pratique. 


\section{NOTES}

1. Sylvain Maresca (Basculer dans le numérique. Les mutations du métier de photographe, 2014) s'est particulièrement intéressé aux effets du passage au numérique sur les conditions d'exercice du métier de photographe. Nous avons rendu compte de son livre dans le numéro 2 de cette revue.

2. Il s'agit certainement d'une des plus belles photos réalisées par Gérald Bloncourt; elle accompagne le grand entretien que nous avions réalisé avec lui et publié dans le numéro 2 de la revue.

3. Cette photo aussi accompagne le grand entretien que Gérald Bloncourt avait accordé à la revue, publié dans son numéro 2 .

\section{AUTEURS}

\section{HENRI ECKERT}

Professeur de sociologie à l'Université de Poitiers et membre du GRESCO. 